Ann. Biol. anim. Bioch. Biophys., I969, 9 (4), 555-562.

\title{
CATABOLISME POST MORTEM DES NUCLEOTIDES ACIDO-SOLUBLES LIBRES ET LIÉS DU MUSCLE DE BOVIN
}

\author{
C. VALIN et J. CHARPENTIER \\ avec la collaboration technique de Anne-Marie Gueugneau et A. OBLed \\ Station de Recherches sur la Viande, \\ Centre de Recherches de Clermont-Ferrand 63 -Saint-Genès-Champanelle \\ Institut national de la Recherche agronomique
}

\section{SOMMAIRE}

La dégradation posthmortem des nucléotides acido-solubles du muscle de Bovin a été étudiée au cours d'une période de conservation de 15 jours à $+4^{\circ} \mathrm{C}$.

Entre I jour et I 5 jours post mortem $50 \mathrm{p}$. Ioo des nucléotides acido-solubles sont déphosphorylés. L'IMP est le composé majeur subissant cette dégradation et les produits de son catabolisme, l'inosine et l'hypoxanthine s'accumulent dans le muscle.

L'ADP lié qui représente la majeure partie, voire la totalité de l'ADP du muscle, n'est pas déphosphorylé au cours de la période étudiée.

\section{INTRODUCTION}

Le catabolisme des nucléotides acido-solubles du muscle joue un rôle important au cours de l'évolution post morten du muscle et de l'élaboration des qualités organoleptiques de la viande. L'hydrolyse de l'A'TP dans les instants suivant l'abattage détermine l'apparition de la rigor mortis et l'accumulation d'IMP dans le muscle (BENDALL, I960).

Les conditions dans lesquelles s'effectue le catabolisme de l'ATP dans la phase de pre rigor peuvent entraîner des modifications profondes des protéines myofibrillaires et provoquer une dénaturation de ces fractions, altérant les qualités organoleptiques de la viande, comme l'illustre l'exemple des viandes exsudatives de porc (BRISKEY, I964). 
La phase de maturation, au cours de laquelle la tendreté augmente et la saveur s'élabore, s'accompagne de la déphosphorylation de l'IMP, formé lors de l'installation de la rigor mortis et de l'apparition de ses produits de dégradation : l'inosine et l'hypoxanthine.

Très étudié dans le cas de l'autolyse de la chair de poisson (JONES et MURRAY, I960-I964) le catabolisme post mortem des nucléotides, en particulier lors de la phase de maturation, n'a pas fait l'objet de recherches systématiques au cours de la transformation du muscle en viande, mise à part l'étude de TERASAKI et al. (I965) sur le muscle de Poulet et de Porc.

La présente publication rapporte, dans le cas du muscle de Bovin, l'évolution des formes libres et liées des nucléotides acido-solubles ainsi que l'apparition de leurs produits de dégradation au cours de la maturation dans les conditions classiques de conservation à $+4^{\circ} \mathrm{C}$.

\section{MATÉRIEL, ET MÉTHODES}

\section{Matériel}

L'étude a porté sur des vaches de réforme non taries, de toutes races et d'un âge variant de 7 à Io ans. L'abattage a été effectué à l'abattoir du C. N. R. Z. de Jouy.

Les carcasses ont été mises à " ressuyer " pendant 24 heures à température ambiante puis placées pendant 15 jours en chambre froide à $+4^{\circ} \mathrm{C}$.

Les déterminations ont porté sur le muscle Longissimus dorsi prélevé 24 heures, 8 jours et 15 jours post mortem au niveau respectivement des $\mathrm{II}^{\mathrm{e}}, \mathrm{I}_{2}{ }^{\mathrm{e}}$ et $\mathrm{I} 3^{\mathrm{e}}$ côte.

\section{Méthodes}

\section{Extraction des nucléotides acido-solubles totaux.}

Toutes les opérations d'extraction ont été effectuées à une température inférieure ou égale à $+4^{\circ} \mathrm{C}$.

Trente grammes de muscle sont homogénéisés au Biorex dans $120 \mathrm{ml}$ de TCA 10 p. 100 (W/V) puis centrifugés à $2000 \mathrm{~g}$ pendant $\mathrm{I} 5$ minutes. Le culot de centrifugation est lavé deux fois dans $5 \circ \mathrm{ml}$ de TCA Io p. I00. Les trois surnageants sont ensuite réunis et filtrés sur filtre Durieux sans cendre. Le TCA est extrait à l'éther sulfurique (V/V) jusqu'à obtention d'un $\mathrm{pH}$ égal à 3,7. L'éther est chassé par barbotage d'azote.

Extraction des nucléotides liés aux protéines myofibrillaires.

Les protéines myofibrillaires sont extraites de $30 \mathrm{~g}$ de muscle homogénéisés dans 7 volumes de $\mathrm{KCl} 0,6 \mathrm{M} ; \mathrm{CO}_{3} \mathrm{HNa} 0,04 ; \mathrm{CO}_{3} \mathrm{Na}_{2}$ o,०I $\mathrm{M}, \mathrm{pH} 9,2$ et purifiées par deux cycles de précipitation et redissolution dans $\mathrm{KCl}$ 0,6 M tamponné à $\mathrm{pH}$ 7,2. L'actomyosine en solution dans du $\mathrm{KCl} 0,6 \mathrm{M}$ est précipitée par dilution, centrifugée et la protéine précipitée est mise à dialyser pendant $\mathrm{i} 6 \mathrm{~h}$ contre 2 fois 50 volumes d'eau distillée.

Après dialyse les nucléotides liés sont obtenus après dénaturation de l'actomyosine par addition de TCA Io p. Ioo selon une technique identique à celle utilisée dans le cas des nucléotides totaux.

\section{Fractionnement des nucléotides acido-solubles.}

La chromatographie des nucléotides acido-solubles a été effectuée selon une adaptation simplifiée de la technique de Hurlbert et PotTer (r954).

Le fractionnement des nucléotides acido-solubles a été obtenu par chromatographie sur colonne de résine Dovex I $\times 8$ (200-400 mesh) sous forme $\mathrm{H} \mathrm{COO}^{-}$. La chromatographie est effectuée à température ambiante sur des colonnes de $30 \mathrm{~cm} \times 1 \mathrm{~cm}$ chargées de 2000 à 2500 DO à $260 \mathrm{m \mu}$ (DO/mi $\times$ volume). L'éluat a été enregistré en continu à $254 \mathrm{~m} \mu$ à l'aide d'un enregistreur Uvicord LKB. L'élution est obtenue par une suite de gradients exponentiels acide formique-formiate. Dans le cas des nucléotides liés un gradient exponentiel d'acide formique o-4 $\mathrm{N}$ est utilisé. 
Pour la détermination quantitative des fractions éluées les contenus des pics sont mesurés au spectrophotomètre Beckman DU à 4 longueurs d'onde, 260, 275, 290 et $310 \mathrm{~m} \mu$. La détermination quantitative des nucléotides, nucléosides et bases a été effectuée en utilisant les coefficients d'extinction molaire donnés par ChargafF et Davidson (1955). Les rapports $\frac{\mathrm{DO} 275}{\overline{\mathrm{DO}} 260}$ et $\frac{\mathrm{DO} 290}{\mathrm{DO} 260}$ après soustraction de l'absorption non spécifique à 3 lo $\mathrm{m} \mu$ permettent d'identifier chaque nucléotide et de juger de la qualité de la séparation.

La technique chromatographique utilisée ne permet pas de séparer le NAD du CMP dont les quantités globales seront exprimées en p. roo de l'extrait TCA total.

Les nucléosides et les bases ne sont pas retenus sur la colonne de Dowex équilibrée sous la forme $\mathrm{H} \mathrm{COO}-$. Cette fraction a été étudiée quantitativement par fractionnement sur Sephadex selon deux méthodes, d'une part sur Sephadex G 25 en milieu bicarbonate $0,02 \mathrm{M}$ et d'autre part par adsorption sur Sephadex G io selon la méthode de SweEtman Nyнam (ig68).

\section{RÉSULTATS ET DISCUSSION}

\section{Nucléotides totaux}

La figure I montre un chromatogramme type des nucléotides acido-solubles totaux du muscle Long Dorsal de Bovin extraits un jour post mortem. Les principaux pics correspondent à des composés ou des dérivés de l'adénine. Une bonne séparation

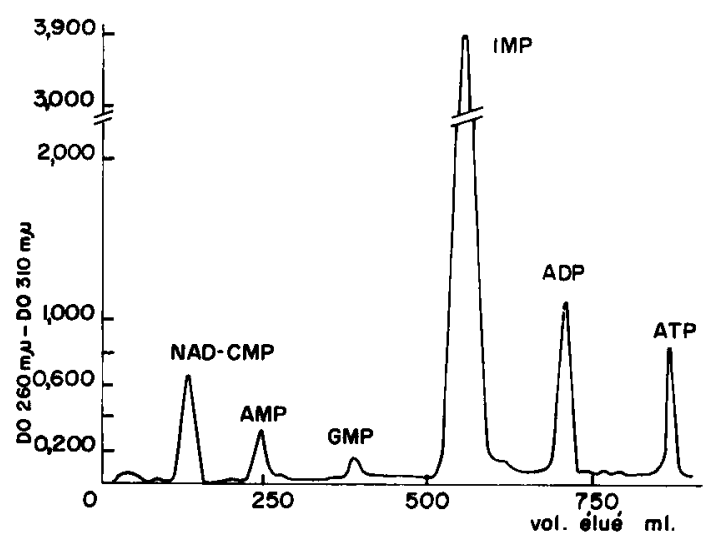

Fig. I. - Chromatographie sur Dozvex $1 \times 8$ des nucléotides acido-solubles totaux du muscle Long Dorsal de Bovin

de 1'AMP, IMP, ADP, ATP et GMP est réalisée par cette technique qui, par ailleurs, ne permet pas de fractionner le CMP du NAD élués simultanément en tête de la chromatographie. Cette première fraction, non négligeable un jour post mortem diminue considérablement au cours de la période de conservation envisagée ainsi que 1'indique le tableau $\mathrm{I}$.

Le tableau 2 rapporte l'évolution subie par les autres fractions au cours de la même période. Ainsi que l'indique ce tableau, à un jour post mortem l'ATP a été presque entièrement hydrolysé dans nos conditions expérimentales. L'AMP et le GMP ne représentent, quantitativement, que des fractions mineures. Enfin deux composés importants l'IMP et l'ADP se caractérisent par une évolution différente, le premier est déphosphorylé lentement entre le $\mathrm{I}^{\mathrm{er}}$ et le $\mathrm{I} 5^{\mathrm{e}}$ jour alors que le second 
semble pratiquement stable au cours de la même période. L'ADP, lié aux protéines myofibrillaires, échappe momentanément à la déphosphorylation que subit 1'ADP provenant de l'hydrolyse de l'ATP dans les premières heures post mortem.

TABLEAU I

Evolution subie par le mélange NAD-CMP au cours de la maturation

\begin{tabular}{|c|c|c|c|}
\hline \multirow{2}{*}{ Animaux } & \multicolumn{3}{|c|}{$\begin{array}{c}\text { Mélange NAD-CMP } \\
\text { en p. } 100 \text { de l'extrait TCA total }\end{array}$} \\
\hline & 1 jour & 8 jours & 15 jours \\
\hline 1 & 3,6 & 2,9 & \\
\hline 2 & 10,6 & 2,0 & \\
\hline 3 & 6,4 & 2,0 & \\
\hline 4 & 8 & 2,3 & \\
\hline 5 & 11,3 & 5 & 1,05 \\
\hline i & 3,9 & 1,0 & 0,3 \\
\hline 7 & 4,2 & 0,7 & 0,4 \\
\hline 8 & 11,7 & 3,1 & 0,6 \\
\hline 9 & 9,6 & 4,0 & 1,6 \\
\hline
\end{tabular}

TABLEAU 2

Nucléotides acido solubles totaux en $\mu$ moles/g muscles frais

\begin{tabular}{l|l|l|l|l|l|l|l|l|l|l|l|l|l|l}
\hline \hline & \\
\hline
\end{tabular}


Il n'a pas été possible au cours de ce travail de mettre en évidence la présence d'IDP ce qui corrobore les résultats de TERASAKI et al. (I965), 1'IMP apparu pouvant donc se former suivant la chaîne :

$$
\mathrm{ATP} \longrightarrow \mathrm{ADP} \longrightarrow \mathrm{AMP} \longrightarrow \mathrm{IMP}
$$

Les produits ultimes de déphosphorylation, nucléosides et bases constituent le non retenu de la chromatographie sur Dowex. La détermination de cette fraction permet une mesure globale du catabolisme post mortem des nucléotides acidosolubles du muscle. Cette fraction croît dans de larges proportions au cours de la maturation comme l'indique le tableau 3 qui permet de constater que $I_{5}$ jours post

TABLEAU 3

Détermination globale des produits de déphosphorylation des nucléotides acido solubles au cours de la maturation

\begin{tabular}{|c|c|c|c|}
\hline \multirow{2}{*}{ Animaux } & \multicolumn{3}{|c|}{$\begin{array}{l}\text { Nucléosides et bases } \\
\text { en p. } 100 \text { de l'extrait TCA total }\end{array}$} \\
\hline & 1 jour & 8 jours & 15 jours \\
\hline 1 & 14,4 & 24,3 & \\
\hline 2 & 9 & 23,1 & \\
\hline 3 & 12,6 & 24,2 & \\
\hline 4 & 12,2 & 33,5 & \\
\hline 5 & 6,5 & 27 & 39,3 \\
\hline 6 & 19,2 & 33,3 & 48,2 \\
\hline 7 & 13,8 & 29,4 & 41,2 \\
\hline 8 & 10,6 & 33,1 & 47,7 \\
\hline 9 & 7,2 & 25,6 & 47,5 \\
\hline
\end{tabular}

mortem près de $50 \mathrm{p}$. Ioo des nucléotides totaux ont été déphosphorylés. Les produits du catabolisme de l'IMP, l'inosine et l'hypoxanthine, sont les constituants majeurs de cette fraction comme 1'indique la figure 2 qui représente un chromatogramme

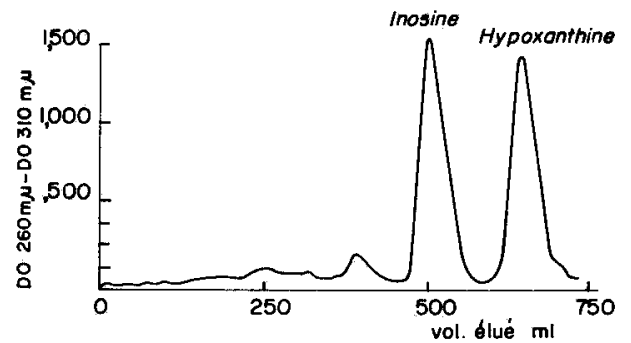

FIG. 2. - Chromatographie sur Sephadex $G 25$ des produits de déphosphorylation des mucléotides acido-solubles du muscle de bovin

sur Sephadex G 25 du non retenu sur Dowex I $\times 8$. Quantitativement la somme de l'inosine plus 1'hypoxanthine peut représenter jusqu'à $95 \mathrm{p}$. Ioo du total du non retenu. La chromatographie sur Sephadex G ro, tout en améliorant la résolution des deux pics conduit à des résultats analogues. 
Le tableau 4 rapporte les quantités d'inosine et d'hypoxanthine, apparues au cours de la maturation, la détermination de ces deux composés est obtenue après chromatographie sur Sephadex.

TABLEAU 4

Détermination quantitative des produits du catabolisme de l'acide inosinique au cours de la maturation

\begin{tabular}{l} 
Inosine et hypoxanthine en $\mu$ moles/g tissu frais \\
\hline Animaux $n^{\text {os }}$ \\
\cline { 2 - 4 }
\end{tabular}

Le bilan après 15 jours de maturation permet de constater que la somme des quantités d'acide inosinique et des produits de son catabolisme correspond pratiquement à la concentration de l'ATP in vivo.

\section{Nucléotides liés}

Au cours d'une période de maturation de $I_{5}$ jours le taux d'ADP ne décroît pas ainsi que l'indique le tableau 2. Il s'agit donc d'une fraction qui échappe momentanément à la déphosphorylation et à la désamination qui caractérisent le catabolisme des nucléotides d'adénine. Les différents travaux concernant les nucléotides liés à l'actine ont tous conclu à la présence d'une mole d'ADP par unité de G actine c'est-à-dire pour $45000 \mathrm{~g}$ d'actine (Tsubor, I968). Compte tenu que l'actine représente $20 \mathrm{p}$. IOO des protéines myofibrillaires cela conduit à une concentration d'ADP de $0,440 \mu$ moles/g de muscle frais ce qui correspond à la valeur moyenne des résultats du tableau 2. Ceci conduit à penser que l'ADP du muscle est pratiquement intégralement représenté par 1'ADP lié aux protéines myofibrillaires.

La détermination après chromatographie sur Dowex (fig. 3) des nucléotides liés à l'actomyosine extraite 8 jours et $\mathrm{I} 5$ jours post mortem sur les 4 derniers animaux a conduit aux résultats du tableau 5 .

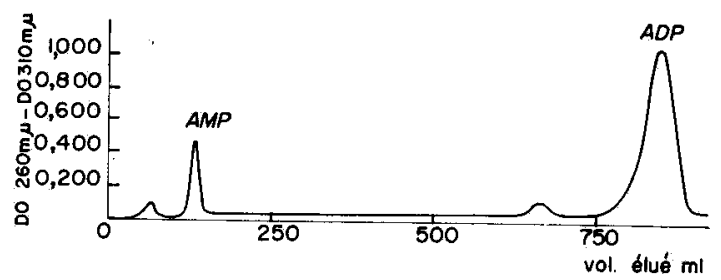

FiG. 3. - Chromatographie sur Dowex $1 \times 8$ des nucléotides liés autx protéines myofibrillaires

Ces résultats indiquent une très faible variation de la quantité d'ADP liée à l'actomyosine extraite 8 jours et I5 jours post mortem, période au cours de laquelle la solubilité de ces protéines évolue peu. 
Le rôle joué par 1'ADP lié à l'actine n'est pas entièrement élucidé. L'implication du nuléotide dans la liaison entre les $\mathrm{G}$ actines constituant les filaments fins dans la structure myofibrillaire est peu vraisemblable (BAIIIN et BARANY, I967 ;

TABLEAU 5

Nucléotides liés aux protéines myofibrillaires

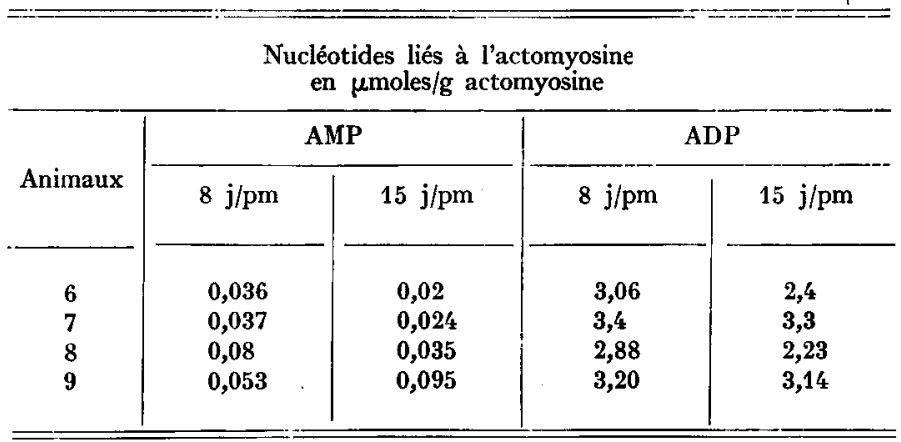

Barány et al., I966). Par contre, la présence de l'ADP fixé sur la $\mathrm{G}$ actine semble indispensable au maintien de sa structure, la dénaturation de la $\mathrm{G}$ actine comme de 1a $\mathrm{F}$ actine intervenant très rapidement avec l'enlèvement de l'ADP (F. OosAwA et al., I965). De plus ni la $\mathrm{G}$ actine ni la $\mathrm{F}$ actine dénaturées ne peuvent fixer del'ADP (SEIDEL et al., I967). TERASAKI et al. (I965) ont observé dans le cas du muscle de Porc un maintien du niveau d'ADP pendant six jours. Par contre dans le cas de myopathie exsudative entraînant une dénaturation importante des protéines myofibrillaires le taux d'ADP s'effondre dans les heures suivant l'abattage (CHARPENTIER, I968).

Au cours de la maturation du muscle de Bovin des remaniements doivent intervenir au niveau de la structure myofibrillaire comme l'indique l'évolution des propriétés de solubilité des protéines qui constituent ces structures (VALIN, I968). Parallèlement, le maintien du taux d'ADP lié, ainsi que l'évolution de certaines propriétés physico-chimiques des protéines myofibrillaires (PENNY, I968) semblent indiquer que ces protéines ne subissent pas de dénaturation importante. Ceci corrobore les premiers résultats d'étude en microscopie électronique (FuKAZAwA-YASUI, I967; VAIIN, I967) qui ne révélaient qu'une destruction de la configuration de la strie $z$ sans que par ailleurs 1'intégrité des myofilaments semble atteinte au cours de cette période post mortem.

\section{CONCLUSION}

L'étude des nucléotides acido-solubles totaux montre que dans la période de I5 jours succédant à l'abattage l'hydrolyse ne dépasse pas $50 \mathrm{p}$. Ioo du taux initial ce qui traduit un catabolisme lent qui s'accompagne d'une faible teneur en hypoxanthine dont le taux reste inférieur à celui de l'inosine. La quantité d'IMP présent dans le muscle à quinze jours post mortem est encore importante et à ce moment la somme de l'IMP et de l'ADP représente pratiquement la totalité des nucléotides acidosolubles du muscle. 
La détermination de l'ADP semble pouvoir constituer une bonne indication de l'état de dénaturation des protéines myofibrillaires. Ce point est intéressant et peut constituer un apport dans l'étude de l'évolution post mortem des protéines myofibrillaires au cours de laquelle il est difficile de faire la part des phénomènes de dénaturation et de 1'hydrolyse enzymatique.

\section{Reçu pour publication en mai 1969.}

\section{SUMMARY}

\section{" POST MORTEM " CATABOLISM OF FREE AND BOUND ACID-SOLUBLE} NUCLEOTIDES IN THE BOVINE MUSCLE

The post mortem degradation of acid-soluble nucleotides in the bovine muscle was studied during a storage period of 15 days at $4^{\circ} \mathrm{C}$.

Within this 15 day period, 50 per cent of acid-soluble nucleotides were dephosphorylated. IMP undergoes the greater degradation (average rates 5.I, $\mu$ moles per gram of fresh muscle at day $\mathrm{I}$, versus $3.35 \mu$ moles per gram at day $\mathrm{I}_{5}$ ). The products of catabolism, $i$. $e$. inosine and hypoxanthine gather up in the muscle.

Bound ADP, which almost entirely constitutes total muscular ADP, within the period considered, was not dephosphorylated.

\section{RÉFÉRENCES BIBLIOGRAPHIQUES}

Bailin G., Baramy M., 1967. Studies on actin-actin and actin-myosin interaction. Biochim. Biophys. acta, 140, 208-22I.

Baramy M., Tucci A. F., Conover T. E., ig66. The removal of the bound ADP of Factin. J. Mol. Biol., 19, 483-502.

Bendall. J. R., I960. post mortem changes in muscle in structure and function of muscle. Edited by G.H. Bourne. Acad. Press., Vol. III, 227-274.

BRISKEY E. J., I964. Etiological status and associated studies of pale soft exsudative porcine musculature. Adv. in Food Res., 13, 89-1 78 .

Chargaff E., Davidson J. N., I955. The Nucleic acids. Vol. I. Acad. Press Inc. Publ. New York.

Charpentier J., i968. Glycogenolyse post mortem du muscle Longissimus dorsi de porc. Ann. Zootech. 17, 429-443.

Fukazowa T., Yasur T., I967. The change in zigzag configuration of the $Z$ line of myofibrils. Biochem. Biophys. Acla, 140, 534-537.

Hurlbert R. B., Schmitz H., Brumm A. F., Potter R., I954. Chromatographic separation of acid soluble nucleotides. J. Biol. Chem., 209, 23-39.

Jones N. R., Murray J., 1960. The acid soluble nucleotides of codling muscle. Biochim. J., 77, 567574.

JoNES N. R., MURRAY J., 1964. Rapid measure of nucleotide dephosphorylation in iced fish muscle. Their value as indices of freshness and IMP concentration. J. Sci. Food Agr., 15, 684-69o.

Oosawa F., Asakura S., Higashi S., Kasai M., 1965. Morphogenesis and motility of actin polymers. In Molecular Biology of muscular contraction. Elsevier Publ. Comp., 56-76.

Penny I. F., 1968. Effect of ageing on the properties of myofibrils of rabbit muscle. J. Sci. Food Agr. 19, 518-524.

Seider D. T., Von Clark D., Weber H. H., 1967. Die Absoluten Affinitäts konstanten von G actin und F actin mit ATP, ITP, CTP, GTP, ADP, and IDP. Biochem. Biophys. acta., 140, 93-I08.

Sweetman L., Nyhan W. L., Ig68. Sephadex G Io adsorption chromatography of purines and related compounds. J. Chromatog., 32, 662-675.

Terasaki M., et al., 1965. Studies on the flavor of meats. Part I. Formation and degradation of inosinic acids in meats. Agr. Biol. Chem., 29, 208-2I5.

Tsubor K. K., I968. Actin and bound nucleotide stœechiometry. Biochim. Biophys. Acta, 160, $420-434$.

Valin C., 1967. Évolution post mortem des protéines myofibrillaires du muscle de Bovin. 13th Europeau meeting of meat research workers. Rotterdam.

VALIN C., I968. post mortem changes in myofibrillar protein solubility. J. Food Tech., (3), I71-I73. 